\title{
Glycyrrhizin Ameliorate Ischemia Reperfusion Lung Injury through Downregulate TLR2 Signaling Cascade in Alveolar Macrophages
}

\author{
Lin Fei ${ }^{1+}$, Feng Jifeng ${ }^{2 t}$, Wang Tiantian ${ }^{1}, \mathrm{He} \mathrm{Yi}^{1}$ and Pan Linghui ${ }^{1 *}$ \\ ${ }^{1}$ Department of Anesthesiology, Affiliated Tumor Hospital of Guangxi Medical University, Nanning, China, ${ }^{2}$ Department of \\ Anesthesiology, Guangxi Maternal and Child Health Hospital, Nanning, China
}

OPEN ACCESS

Edited by:

Narasaiah Kolliputi,

University of South Florida,

United States

Reviewed by:

Zhongqiu Xie,

University of Virginia, United States

Sunil Kumar Panigrahi,

Columbia University, United States

Bibhuti Mishra,

University of Massachusetts Medical

School, United States

*Correspondence:

Pan Linghui

flylin0208@163.com;

28144273@qq.com

†These authors have contributed equally to this work.

Specialty section: This article was submitted to Respiratory Pharmacology, a section of the journal

Frontiers in Pharmacology

Received: 18 April 2017 Accepted: 02 June 2017

Published: 16 June 2017

Citation:

Fei L, Jifeng F, Tiantian W, Yi H and Linghui P (2017) Glycyrrhizin Ameliorate Ischemia Reperfusion Lung Injury through Downregulate TLR2 Signaling Cascade in Alveolar Macrophages.

Front. Pharmacol. 8:389 doi: 10.3389/fphar.2017.00389
This experiment was conducted to study whether pretreatment with Glycyrrhizin (GL) could ameliorate ischemia-reperfusion (I/R) lung injury and investigate the mechanisms of its protective effects in a mice model. Six-eight weeks male BALB/C mice were randomly assigned to four groups $(n=6)$ : Control, Glycyrrhizin, I/R and I/R+Glycyrrhizin. Lung I/R was achieved by clamping the left hilus pulmonis. GL (200 mg/kg) was injected intraperitoneally 30 min before anesthesia. Measurement of pathohistological lung injury score, pulmonary permeability, isolated alveolar macrophages, inflammatory mediators, TLR2 and its downstream factors (MyD88, NF-kB) were performed. The results were as anticipated. Pathohistological evaluation indicated that GL significantly ameliorated I/R-induced lung injury, pulmonary permeability and edema. Pretreatment with GL significantly inhibited I/R-induced inflammation in lung tissues and BALF. In addition, GL significantly decreased I/R-induced isolated alveolar macrophages and suppressed I/R-induced expression of TLR2 and its downstream factors in lung tissues and alveolar macrophages. Collectively, our data indicated that pretreatment with GL could ameliorate I/R lung injury. The mechanisms of its protective effects might be inhibit I/R-induced inflammatory response through downregulate TLR2 signaling cascade in alveolar macrophages.

Keywords: ischemia reperfusion lung injury, Glycyrrhizin, TLR2, alveolar macrophages, inflammatory mediators

\section{INTRODUCTION}

Lung ischemia-reperfusion injury (LIRI) can occur in lung transplantation, lung resection, cardiac arrest, and pulmonary emboli. Lung dysfunction caused by LIRI is a strong risk factor for acute lung injury (ALI), and portends worse patient outcomes (Fiser et al., 2002; de Perrot et al., 2003; Weyker et al., 2013). Inflammatory response has significant role in tissue damage after organ ischemiareperfusion (I/R) (Gelderblom et al., 2012; Diamond et al., 2013; Phelan et al., 2015). Recent studies suggest that $\mathrm{I} / \mathrm{R}$ injury could lead to sterile inflammation through activating the innate immune system. Characterization of the cell-specific sterile inflammatory events in LIRI is a key step toward treatment strategies aimed at alleviating LIRI and improving patient outcomes (Lin et al., 2011; Prakash et al., 2015; Salameh and Dhein, 2015). 
Toll-like receptors (TLRs) represent an conserved family of pattern recognition receptors. TLRs in alveolar macrophages have ability to recognize endogenous ligands and play a pivotal role in innate immunity and sterile inflammation. The ability to initiate inflammatory response makes them suitable candidates for early signaling in LIRI (Miyake, 2007; Lin et al., 2011; Zheng et al., 2013). TLR2 has been implicated as a crucial modulator in $\mathrm{I} / \mathrm{R}$ models and has an important role in the initiation of inflammation during I/R process. Using deletion or pharmacologic antagonism of TLR2 can reduce injury severity in lung, renal, and cerebral models of ischemia reperfusion (Favre et al., 2007; Shigeoka et al., 2007; Phelan et al., 2015). TLR2 signal is required for recruiting Myeloid differentiation factor 88 (MyD88) to the receptors. These kinases ultimately activate transcription factors such as nuclear factor- $\kappa \mathrm{B}(\mathrm{NF}-\kappa \mathrm{B})$ which result in production of various proinflammatory cytokines. However, the cellular and molecular pathways responsible for sterile inflammatory events in LIRI have not been fully delineated. How to control the activation of TLR2 signaling pathway in process of $\mathrm{I} / \mathrm{R}$ lung injury is an important issue to exploration.

Glycyrrhizin (GL), a triterpenoid saponin compound, is the main constituent in the roots and rhizomes of licorice (Glycyrrhiza glabra) and has anti-viral and anti-inflammatory effects. Several studies have shown that GL could attenuate the activation of TLRs and reduce macrophage cytokine release (Yang et al., 2010; Chang et al., 2014). This effect of GL could be a possible way to widen the therapeutic window of I/R injury (Zhang et al., 2014). However, few studies have been designed to investigate its use in a LIRI model.

The purpose of this study was to investigate the protective effects of GL in LIRI mice model and seek to explore the molecular mechanism responsible for its effects.

\section{MATERIALS AND METHODS}

\section{Animal Groups}

The animal protocol was approved by the Institutional Animal Care and Use Committee of Guangxi Medical University (Nanning, China). All animal studies were carried out in accordance with the animal's guidelines of the University Institutional Animal Care and Use Committee. Six-eight weeks male BALB/C mice $(30 \pm 2$ g, Animal Centre, Guangxi Medical University, China) were randomly assigned to four groups: (1) Control group (not being exposed to anesthesia or surgery or any drugs), (2) Glycyrrhizin group (only injected intraperitoneally Glycyrrhizin), (3) I/R group (lung ischemia-reperfusion), (4) I/R+ Glycyrrhizin group.

\section{Glycyrrhizin Application}

Glycyrrhizin acid ammonium salt (catalog number: sc-203059; Santa Cruz, CA, United States) was dissolved in acetic acid and injected intraperitoneally at $200 \mathrm{mg} / \mathrm{kg}$ at $30 \mathrm{~min}$ before anesthesia. The dose and usage of GL were chosen based on previous study (Kim et al., 2015).

\section{Animal Model of LIRI}

Mice were anesthetized by intraperitoneal injection of $10 \%$ chloral hydrate $(4.5 \mathrm{~mL} / \mathrm{kg})$. Next, mechanical ventilation was applied using a RSP1002-type small animal ventilator with a respiratory ratio (inspired to expired air) of $1: 1$ and a breathing frequency 80 breaths $/ \mathrm{min}$. The tidal volume was $10 \mathrm{~mL} / \mathrm{kg}$ and the fraction of inspired oxygen was $100 \%$. Lung collapse and expansion were observed. The mice in I/R group had underwent thoracotomy, occluded the left hilus pulmonis (including pulmonary artery, vein, and bronchi) by a microvascular clamp for $60 \mathrm{~min}$, and followed by $120 \mathrm{~min}$ of reperfusion before closing thoracic incision (Mussi et al., 2008). At $2 \mathrm{~h}$ after operation, mice were sacrificed by cervical vertebra dislocation. Portions of the lower lobe of the left lung were excised from mice for measurement in all groups. No mouse mortality was recorded during the experimental approach.

\section{Hematoxylin and Eosin (H\&E) Staining}

As we described previously (Lin et al., 2015), mice lung tissues were fixed in $4 \%(\mathrm{w} / \mathrm{v})$ paraformaldehyde and embedded in paraffin. Sections were prepared and evaluated by experienced pathologists who were blinded to the experimental treatment conditions. The thickness of the slices (4 microns) were waxed off by xylene and hydration, stained $5 \mathrm{~min}$ by hematoxylin, differentiated $30 \mathrm{~s}$ by hydrochloric acid ethanol, soaked $15 \mathrm{~min}$ in water, stained 2 min by eosin. And then the slices of $\mathrm{H} \& \mathrm{E}$ staining were completed after conventional dehydration, transparent and sealing.

\section{Microscopy and Histology Scoring of Lung Injury}

Hematoxylin and Eosin stained paraffin-mounted lung sections were examined by light microscopy. An investigator who was blinded to the group assignment was assigned to examine the lung $\mathrm{H} \& \mathrm{E}$ slides and determine the levels of lung injury with a scoring system. For each mouse, 10 fields were examined at $200 \times$ total magnification. Scoring was performed as described elsewhere (Prakash et al., 2015). Briefly, the first criterion was infiltration or aggregation of inflammatory cells in air space or vessel walls: $1=$ only wall, $2=$ few cells in air space, $3=$ intermediate, $4=$ severe (air space congested). Second criterion was interstitial congestion and hyaline membrane formation: $1=$ normal lung, $2=$ moderate ( $>25 \%$ of lung section), $3=$ intermediate ( $25-50 \%$ of lung section), $4=$ severe $(>50 \%$ of lung section). Third criterion was hemorrhage: $0=$ absent, $1=$ present.

\section{Determination of Wet/Dry Ratio}

As we described previously (Dai et al., 2015), Pulmonary wet/dry ratios were measured as an index of pulmonary edema and congestion. After mice were killed, the lower lobe of the left lung was immediately weighed and then dried to a constant weight at $60^{\circ} \mathrm{C}$ for $24 \mathrm{~h}$. 


\section{Collection of BALF and Alveolar Macrophages}

Alveolar macrophages were isolated as we described previously (Dai et al., 2015). In brief, the lungs were flushed once with $5 \mathrm{~mL}$ of cold phosphate buffered saline through the cannulated trachea (Dulbecco's PBS; Gibco BRL, Grand Island, NY, United States) to collect bronchoalveolar lavage fluid (BALF). The lungs were subsequently flushed another eight times with $10 \mathrm{~mL}$ PBS to obtain alveolar macrophages.

\section{Pulmonary Vascular Permeability through Total Proteins in BALF}

The level of total proteins in BALF is a marker of alveolar vascular permeability. Determination of total proteins was performed using the bicinchoninic acid (BCA) assay according to the manufacturer's instructions (Pierce, Rockford, IL, United States).

\section{Counting of Alveolar Macrophages}

As we described previously (Dai et al., 2015), BALF was resuspended in Dulbecco's modified Eagle medium (DMEM, Gibco, United States), counted, and transferred to 24-well culture plates (BD, Franklin Lakes, NJ, United States). After incubation for $60 \mathrm{~min}$ at $37^{\circ} \mathrm{C}$ in a $5 \% \mathrm{CO}_{2}$ atmosphere, cultures were washed with DMEM to remove non-adherent cells. The adherent cells were counted using a hemocytometer, viability was determined using a $0.2 \%$ trypan blue exclusion assay, and cell differentiation and aggregation were examined by counting 500 cells on a Wright-Giemsa-stained slide. These cultures also served as the source for analyzing protein expression in alveolar macrophages.

\section{Western Blot Analysis}

The left lung tissues $(50 \mu \mathrm{g})$ and alveolar macrophages $\left(5 \times 10^{6}\right)$ were homogenized in RIPA buffer (catalog number: 89900; Thermo Scientific, Worcester, MA, United States) containing protease inhibitor cocktail (catalog number: P2714; Sigma, St Louis, MO, United States) and phosphatase inhibitor cocktail (catalog number: 04906845001; Roche Applied Science, Indianapolis, IN, United States). Homogenates were centrifuged at $13,000 \mathrm{rpm}$ at $4^{\circ} \mathrm{C}$ for $20 \mathrm{~min}$. The supernatant was collected as the total proteins of lung tissues and alveolar macrophages. The protein concentration was determined by Pierce BCA protein assay kit (catalog number: 23227; Pierce Biotechnology, Rockford, IL, United States). Twenty microgram proteins per lane were separated on a polyacrylamide gel. The proteins were then transferred onto a polyvinylidene difluoride membrane. The membranes were incubated with the following primary antibodies overnight at $4^{\circ} \mathrm{C}$ : rabbit monoclonal anti-TLR2 antibody (1:1000, catalog number: ab108998; Abcam, Cambridge, MA, United States), anti-MyD88 antibody (1:1000, catalog number: ab2064; Abcam, Cambridge, MA, United States), antiNF- $\kappa B$ p105/p50 antibody (1:1000, catalog number: ab32360; Abcam, Cambridge, MA, United States), mouse monoclonal anti- $\beta$-Actin antibody (1:5000, catalog number: ab6276; Abcam, Cambridge, MA, United States). Protein bands were visualized using enhanced chemiluminescence (Pierce, United States). The protein band intensities of TLR2, MyD88, NF- $\kappa B$ proteins were normalized to those of $\beta$-Actin. The results from animals under various experimental conditions then were normalized by mean values of the corresponding control animals.

\section{Lung Tissues and BALF Measurements of IL-1 $\beta$ and IL-6}

ELISA kits for measuring mice IL-1 $\beta$ and IL- 6 (catalog number: MLB00C and M6000B, respectively; R\&D Systems, Minneapolis, $\mathrm{MN}$, United States) were used to quantify the contents of these cytokines in the lung tissues and BALF according to the manufacturer' instructions. The quantity of IL- $1 \beta$ and IL- 6 in the lung tissues and BALF was standardized to the protein contents.

\section{Statistical Analysis}

Parametric results in normal distribution are presented as mean $\pm \mathrm{SD}(n \geq 6)$. All data were analyzed by one-way analysis of variance followed by the Tukey test if the data were normally distributed or by one-way analysis of variance on ranks followed by the Tukey test if the data were not normally distributed. These non-normally distributed data were presented as box plots in the figures. Differences were considered significant at $P<0.05$ based on two-tailed hypothesis testing. All statistical analyses were performed with SigmaStat (Systat Software, Point Richmond, CA, United States).

\section{RESULTS}

\section{GL Ameliorates LIRI in Morphology}

Hematoxylin and Eosin staining and histology scoring of lung tissue were performed to assess the extent of lung injury. Figure 1A showed the results for lung morphology in all groups. In the control and GL groups, lung tissue structures were intact, lacking inflammatory cell infiltration and alveolar wall thickening. In I/R group, alveolar rupture and tissue congestion were observed, the alveolar septa had widened and ambiguous. In I/R+GL group, lung tissue structures were mainly intact, some neutrophils infiltration and red blood cells had leaked into the alveolar space. The lung injury score was significantly higher in I/R group compared with the control and GL group. The levels of lung $\mathrm{I} / \mathrm{R}$ injury were significantly ameliorated in $\mathrm{I} / \mathrm{R}+\mathrm{GL}$ group compared with $\mathrm{I} / \mathrm{R}$ group. No difference was noted between control group and GL group (Figure 1B).

\section{GL Decreases I/R-Induced Pulmonary Permeability and Edema}

Pulmonary wet/dry ratios and the levels of total proteins in BALF were measured to assess pulmonary permeability and edema. Figure 2 presented the levels of total proteins in BALF (Figure 2A) and the wet/dry ratios in lung tissues (Figure 2B). GL significantly reduced I/R-induced increases in the levels of total proteins in BALF $(P<0.05)$ and the wet/dry ratios in lung tissue $(P<0.05)$. 

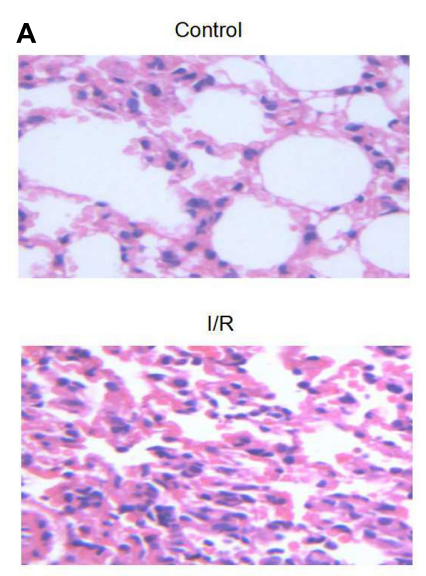
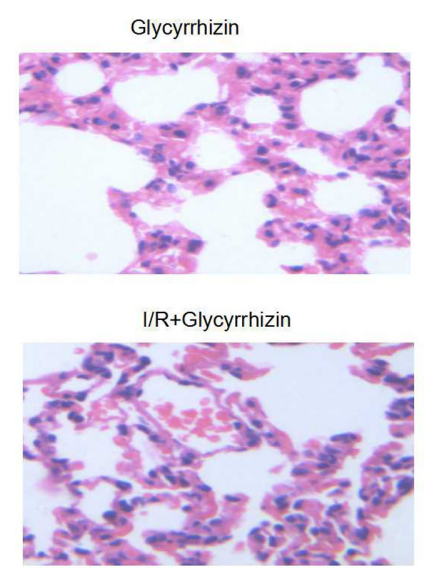

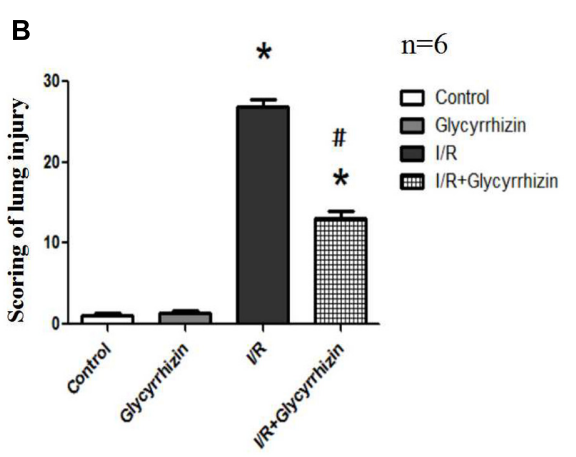

FIGURE 1 | Morphological changes in Control, Glycyrrhizin, I/R and I/R+Glycyrrhizin groups by H\&E staining (200x) (A). The scoring of lung injury shown in (B) ( ${ }^{*} P<0.05$, compared with control. ${ }^{\#} P<0.05$, compared between $\mathrm{I} / \mathrm{R}$ and $\mathrm{l} / \mathrm{R}+$ Glycyrrhizin group).
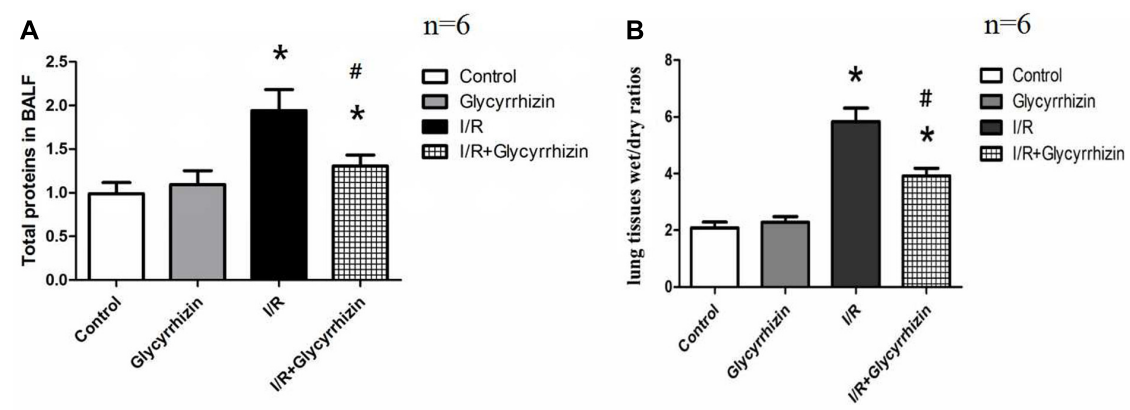

FIGURE 2 | Glycyrrhizin decreases I/R-induced pulmonary permeability and edema. (A) Graphic presentation of Total proteins in BALF. (B) Graphic presentation of the Wet/dry ratios in lung tissues. ( $P<0.05$, compared with control. ${ }^{*} P<0.05$, compared between I/R and I/R+Glycyrrhizin group).

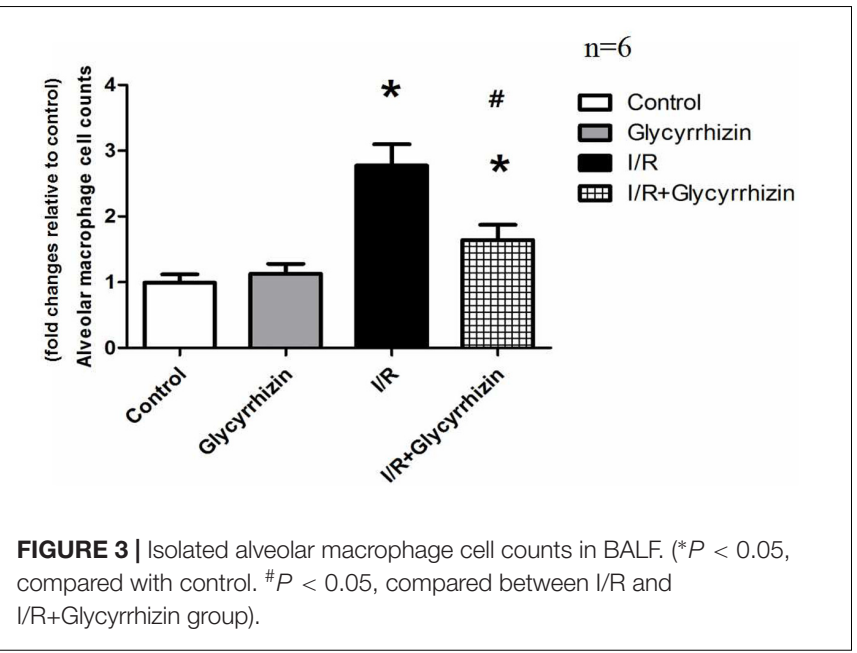

\section{GL Reduces I/R-Induced Isolated Alveolar Macrophages}

Isolated alveolar macrophages were counted to assess the activity of the alveolar macrophages. The percentage of alveolar macrophages was more than $90 \%$ counted by the hemocytometer. Compared with control and GL group, isolated alveolar macrophages in BALF had significantly increased in the I/R group (Figure 3). GL significantly reduced I/R-induced isolated alveolar macrophages.

\section{GL Inhibits I/R-Induced Inflammation in Lung Tissues and BALF}

IL- $1 \beta$ and IL- 6 in lung tissues and BALF were measured to assess the inflammatory response. Figure 4 showed the levels of IL-1 $\beta$ and IL- 6 in lung tissues and BALF. The results demonstrated that I/R injury significantly increased the levels of IL- $1 \beta$ and IL-6 in Lung tissues and BALF compared with the control and GL group $(P<0.05)$. GL significantly inhibited the $\mathrm{I} / \mathrm{R}$-induced IL-1 $\beta$ and IL-6 expression compared with the I/R group $(P<0.05)$.

\section{GL Inhibits I/R-Induced TLR2 Expression in Lung Tissues and Alveolar Macrophages}

Toll-like receptor2 protein expression in Lung tissues and alveolar macrophages were determined by Western Blot Analysis. 

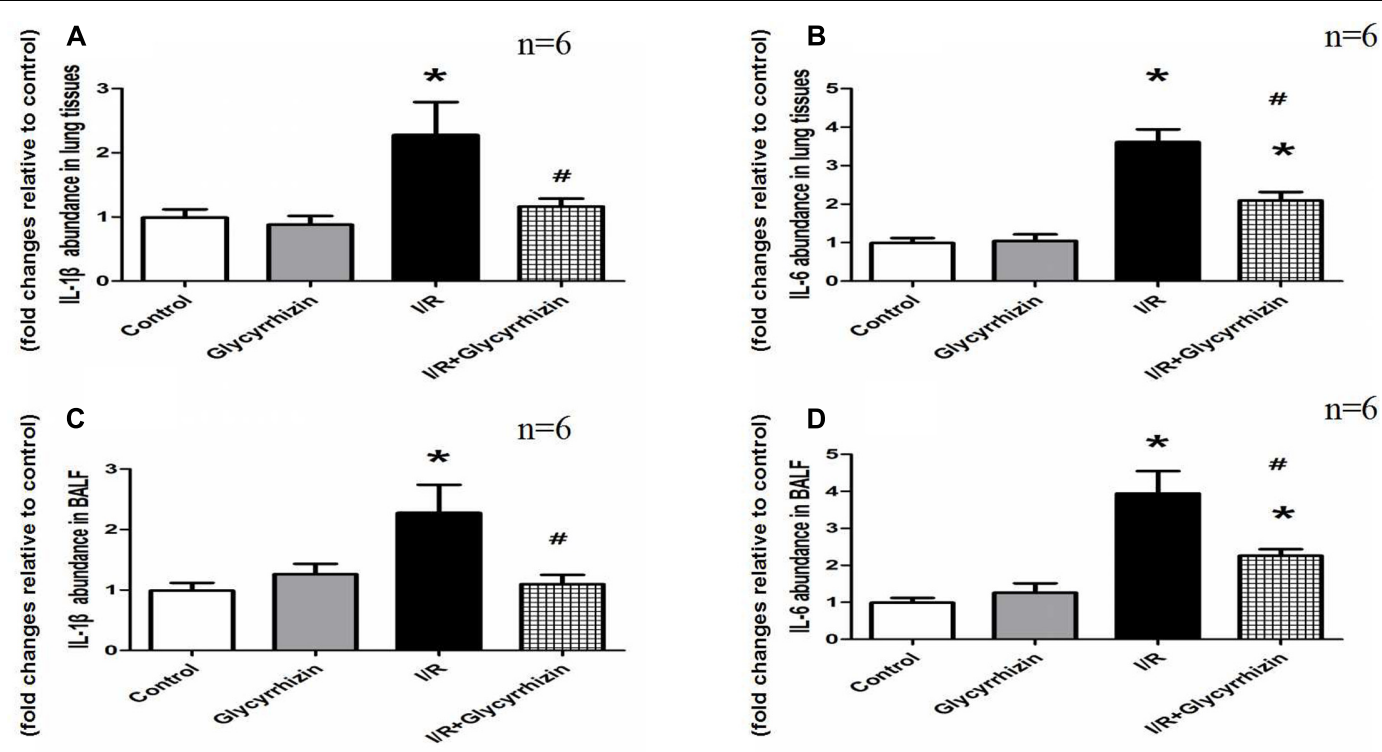

FIGURE 4 | Glycyrrhizin inhibits I/R-induced IL-1 $\beta$ and IL-6 in lung tissues and BALF. (A) Graphic presentation of IL-1 $\beta$ abundance in lung tissues. (B) Graphic presentation of IL-6 abundance in lung tissues. (C) Graphic presentation of IL-1 $\beta$ abundance in BALF. (D) Graphic presentation of IL-6 abundance in BALF. ( $P<0.05$, compared with control. ${ }^{*} P<0.05$, compared between $\mathrm{I} / \mathrm{R}$ and $\mathrm{I} / \mathrm{R}+\mathrm{Glycyrrhizin}$ group).

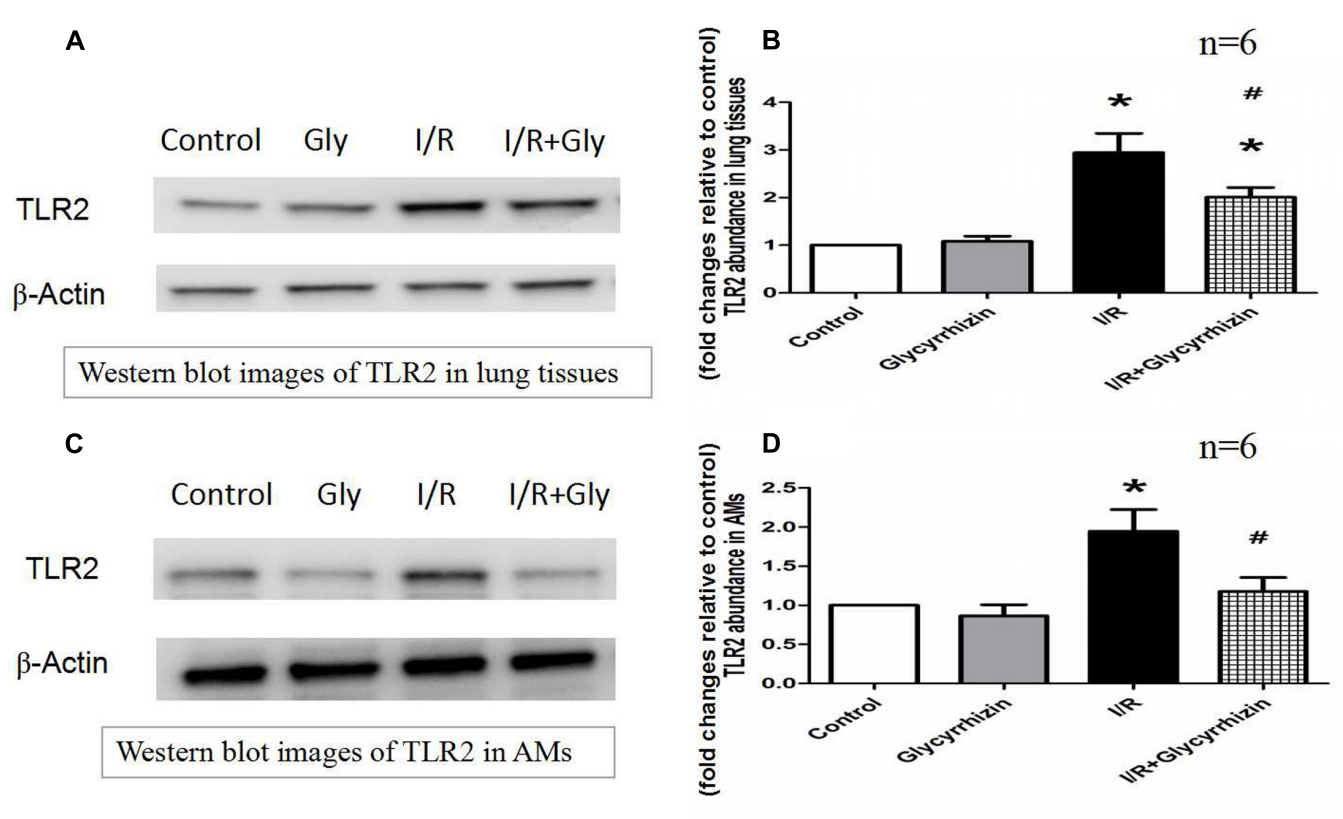

FIGURE 5 | Glycyrrhizin inhibits I/R-induced TLR2 expression in Lung tissues and alveolar macrophages. (A) Representative Western blot images of TLR2 in lung tissues. (B) Graphic presentation of TLR2 abundance in lung tissues. (C) Representative Western blot images of TLR2 in AMs. (D) Graphic presentation of TLR2 abundance in AMs. ( ${ }^{*} P<0.05$, compared with control. ${ }^{\#} P<0.05$, compared between I/R and I/R+Glycyrrhizin group).

The results in Figure 5 demonstrated that I/R injury had significantly increased the expression of TLR2 in lung tissues and alveolar macrophages compared with the control and GL group $(P<0.05)$. GL significantly inhibited the I/Rinduced TLR2 expression compared with the I/R group $(P<0.05)$.

\section{GL Modulates the Expression of Downstream Factors of TLRs in Alveolar Macrophages}

MyD88 and NF- $\kappa$ B protein expression in alveolar macrophages were determined to assess the expression of downstream factors 

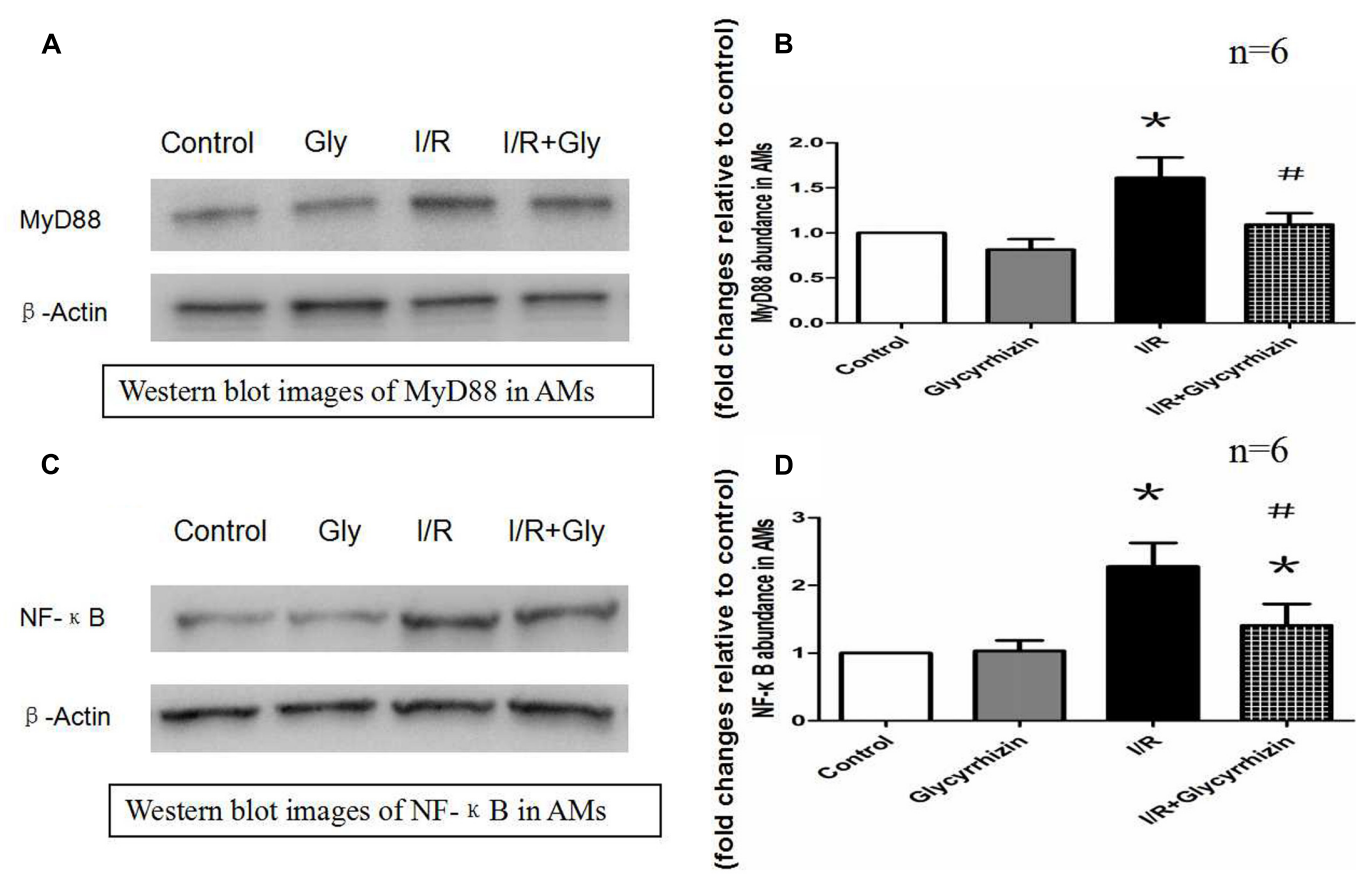

FIGURE 6 | Glycyrrhizin inhibits I/R-induced MyD88 and NF-кB expression in AMs. (A) Representative Western blot images of MyD88 in AMs. (B) Graphic

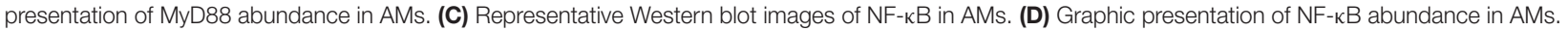
( ${ }^{*} P<0.05$, compared with control. ${ }^{\#} P<0.05$, compared between I/R and I/R+Glycyrrhizin group).

of TLRs. The results in Figure 6 demonstrated that I/R injury significantly increased the expression of MyD88 and NF- $\kappa$ B in alveolar macrophages compared with the control and GL group $(P<0.05)$. The results also illustrated that treatment with $\mathrm{GL}$ significantly reduced MyD88 and NF- $\kappa$ B expression compared with the I/R group.

\section{DISCUSSION}

This study showed that treatment with GL had a strong protective effect in the I/R lung. GL treatment was found to decrease I/R-induced pulmonary permeability and edema and ameliorate the levels of lung I/R injury in morphology. In addition, administration of GL significantly inhibited I/R-induced inflammation and isolated alveolar macrophages. The present results suggest that protective effect of GL in I/R is, at least in part, attributable to its inhibitory effects on TLR2 and subsequently inhibition of TLR2 downstream factors (MyD88 and NF- $\mathrm{B}$ ) in alveolar macrophages.

In this study, we chose $\mathrm{BALB} / \mathrm{c}$ mouse for the animal model because BALB/c mouse had been widely used in animal experiment of immunology and physiology. Recently, a number of studies have indicated that $\mathrm{I} / \mathrm{R}$ injury is characterized by acute local inflammation. During reperfusion after acute ischemia, neutrophils are believed to exacerbate tissue damage by obstruction of vessels, augment of pulmonary permeability and release of proinflammatory cytokines and cytolytic enzymes. However, Why relatively short interruption and reperfusion of blood flow lead to inflammation remains an unresolved question of human physiology. And how to control the process of $\mathrm{I} / \mathrm{R}$ inflammatory response is still an important issue to exploration.

Toll-like receptors refer to biomolecules produced by invading microbes or released from damaged tissue. These biomolecules serve as danger signals that initiate an inflammatory immune response. TLR2 is crucial for PAMP signaling and can work through the adaptor protein MyD88, which activates NF-кB and ultimately stimulates the production of proinflammatory cytokines (Shigeoka et al., 2007; Yang et al., 2010). TLRs trigger inflammation mediated by complement, macrophages, and neutrophils. These cells produce chemokines or cytokines that mediate systemic immune responses and recruit leukocytes to the sites of inflammation (Miyake, 2007; Ding et al., 2017). In the process of systemic immune reaction following exposure to harmful stimulus, alveolar macrophages are the primary producers of proinflammatory cytokines in lungs (Wang et al., 2012). The rapidly activated of alveolar macrophages suggests that they may play a crucial role in the pathogenesis of lung injury. Several previous reports have demonstrated removing alveolar macrophages from rats or mice could attenuate the alveolar barrier dysfunction and inflammatory lung injury (Frank et al., 2006; Eyal et al., 2007).

In our previous study (Dai et al., 2015; Huang et al., 2017), the results indicated that TLR2, TLR4, and TLR9 on alveolar macrophages and release of pro-inflammatory cytokines play a role in ventilator-induced lung injury. In this study, expression of TLR-2 and its downstream factors (MyD88 and NF-кB) in alveolar macrophages were significantly enhanced 
in $\mathrm{I} / \mathrm{R}$ group compared to control group. These results are in accordance with previous other study (Ma et al., 2013) who demonstrated an upregulation of TLR-2, TLR-4, and MyD88 in MCAO stroke model. Moreover, TLR-2 deficiency caused significant reduction of infarct volume and suppressed inflammatory cytokine expression in infiltrating macrophages after brain ischemia (Shichita et al., 2012). Therefore, we believe that alveolar macrophages are rapidly activated, and the expression of TLR2 in alveolar macrophages plays an important role in the initiation of inflammation during the process of lung I/R.

Now the effective and applicable pharmacological treatments for LIRI is still absent. GL is a natural triterpene glycoside that has multiple biological activities, including anti-inflammatory, anti-viral, anti-oxidative, anti-cancerous, immune adjustment, organ-protective activities (Kim et al., 2011; Gong et al., 2012). The organ-protective effects of GL were previously evidenced by HMGB1-TLR4-IL-17A signaling pathway in the postischemic brain (Zhang et al., 2014), inhibition of reactive oxygen spices generation by neutrophils (Asl and Hosseinzadeh, 2008), ameliorating ischemic brain damage through downregulation of the TLR signaling cascade (Barakat et al., 2014). Previously reports also proved that GL has anti-inflammatory effects. In kainic acid-induced seizure animal model, treatment with GL might be attributable to the inhibitions of HMGB1 induction and release, which in turn, mitigates the inflammatory process (Luo et al., 2014). In rat skin thermal injury model, GL might possesses an anti-inflammation effect to protect the remote organs from burn-induced injury (Shen et al., 2015). All these actions make GL might be a suitable candidate for the treatment of LIRI. However, few studies have been designed to investigate its use in a LIRI model. In the current study, GL significantly reduced the lung injury score, decreased I/R-induced pulmonary permeability and isolated alveolar macrophages, inhibited I/R-induced inflammation in lung tissues and BALF. Our results indicated that treatment with GL have antiinflammatory and organ-protective effects in LIRI animal model.

The mechanism by which GL exerted these effects is still unclear. Kim considered that GL had inhibitory effect on oxidative damage and suppress caspase-3 activation (Kim and Lee, 2008). Ogiku et al. (2011) found that GL protected ischemia

\section{REFERENCES}

Asl, M. N., and Hosseinzadeh, H. (2008). Review of pharmacological effects of Glycyrrhiza sp. and its bioactive compounds. Phytother. Res. 22, 709-724. doi: 10.1002/ptr.2362

Barakat, W., Safwet, N., El-Maraghy, N. N., and Zakaria, M. N. (2014). Candesartan and glycyrrhizin ameliorate ischemic brain damage through downregulation of the TLR signaling cascade. Eur. J. Pharmacol. 724, 43-50. doi: 10.1016/j.ejphar. 2013.12.032

Chang, C. Z., Wu, S. C., and Kwan, A. L. (2014). Glycyrrhizin attenuates toll like receptor-2, -4 and experimental vasospasm in a rat model. J. Immunol. Res. 2014:740549. doi: 10.1155/2014/740549

Dai, H., Pan, L., Lin, F., Ge, W., Li, W., and He, S. (2015). Mechanical ventilation modulates Toll-like receptors 2, 4, and 9 on alveolar macrophages in a ventilator-induced lung injury model. J. Thorac. Dis. 7, 616-624. doi: 10.3978/j. issn.2072-1439.2015.02.10 reperfusion liver injury by stabilization of the membrane structure of hepatocytes. In addition, GL was reported to attenuate inflammatory responses induced by TLR- 3 and TLR-4, alter the integrity of the plasma membrane and attenuate receptor mediated signaling (Schröfelbauer et al., 2009). Barakat et al. (2014) found that GL suppressed the expression of TLR-2, TLR-4 expression in brain ischemia. In the present study, our data indicated that treatment with GL suppressed I/R-induced TLR2 expression in Lung tissues and alveolar macrophages and modulated the expression of downstream factors of TLR2. Our results are in accordance with previously reports. Moreover, to our knowledge, this is the first study to indicate the effect of GL on the expression of TLR2 and its downstream factors in alveolar macrophages after LIRI. This could be attributed to a reduction in alveolar macrophages activation with the resultant decrease TLR2 and inflammatory mediators.

\section{CONCLUSION}

Pretreatment with GL could ameliorate I/R lung injury. The mechanisms of its protective effects might be inhibit I/R-induced inflammatory response through downregulate TLR2 signaling cascade in alveolar macrophages.

\section{AUTHOR CONTRIBUTIONS}

LF: Study design, fund collection, samples detection, manuscript preparation. FJ: Data interpretation, samples detection, literature search. WT: Data collection, animal model. HY: Statistical analysis. PL: Study design, fund collection, manuscript preparation.

\section{ACKNOWLEDGMENTS}

The following grants and foundations supported this work: Research grant No. 81460016, 81560018 from the National Natural Science Foundation of China and Research grant No. S201418-05 from the Bureau of Health, Guangxi.

de Perrot, M., Liu, M., Waddell, T. K., and Keshavjee, S. (2003). Ischemiareperfusion-induced lung injury. Am. J. Respir. Crit. Care Med. 167, 490-511. doi: 10.1164/rccm.200207-670SO

Diamond, J. M., Lee, J. C., Kawut, S. M., Shah, R. J., Localio, A. R., Bellamy, S. L., et al. (2013). Clinical risk factors for primary graft dysfunction after lung transplantation. Am. J. Respir. Crit. Care Med. 187, 527-534. doi: 10.1164/rccm. 201210-1865OC

Ding, X., Jin, S., Tong, Y., Jiang, X., Chen, Z., Mei, S., et al. (2017). TLR4 signaling induces TLR3 up-regulation in alveolar macrophages during acute lung injury. Sci. Rep. 7:34278. doi: 10.1038/srep34278

Eyal, F. G., Hamm, C. R., and Parker, J. C. (2007). Reduction in alveolar macrophages attenuates acute ventilator induced lung injury in rats. Intensive Care Med. 33, 1212-1218. doi: 10.1007/s00134-0070651-X

Favre, J., Musette, P., Douin-Echinard, V., Laude, K., Henry, J. P., Arnal, J. F., et al. (2007). Toll-Like receptors 2 -deficient mice are protected against postischemic 
coronary endothelial dysfunction. Arterioscler. Thromb. Vasc. Biol. 27, 1064-1071. doi: 10.1161/ATVBAHA.107.140723

Fiser, S. M., Tribble, C. G., Long, S. M., Kaza, A. K., Kern, J. A., Jones, D. R., et al. (2002). Ischemia-reperfusion injury after lung transplantation increases risk of late bronchiolitis obliterans syndrome. Ann. Thorac. Surg. 73, 1041-1048. doi: 10.1016/S0003-4975(01)03606-2

Frank, J. A., Wray, C. M., McAuley, D. F., Schwendener, R., and Matthay, M. A. (2006). Alveolar macrophages contribute to alveolar barrier dysfunction in ventilator-induced lung injury. Am. J. Physiol. Lung Cell. Mol. Physiol. 291, L1191-L1198. doi: 10.1152/ajplung.00055.2006

Gelderblom, M., Weymar, A., Bernreuther, C., Velden, J., Arunachalam, P., Steinbach, K., et al. (2012). Neutralization of the IL-17 axis diminishes neutrophil invasion and protects from ischemic stroke. Blood 120, 3793-3802. doi: 10.1182/blood-2012-02-412726

Gong, G., Yuan, L. B., Hu, L., Wu, W., Yin, L., Hou, J. L., et al. (2012). Glycyrrhizin attenuates rat ischemic spinal cord injury by suppressing inflammatory cytokines and HMGB1. Acta Pharmacol. Sin. 33, 11-18. doi: 10.1038/aps. 2011.151

Huang, C., Pan, L., Lin, F., Dai, H., and Fu, R. (2017). Monoclonal antibody against Toll-like receptor 4 attenuates ventilator-induced lung injury in rats by inhibiting MyD88 and NF-кB-dependent signaling. Int. J. Mol. Med. 3, 693-700.

Kim, S. W., Lim, C. M., Lee, H. K., and Lee, J. K. (2011). The use of stronger neo-minophagen $\mathrm{C}$, a glycyrrhizin-containing preparation, in robust neuroprotection in the post- ischemic brain. Anat. Cell Biol. 44, 304-313. doi: $10.5115 /$ acb.2011.44.4.304

Kim, Y. J., and Lee, C. S. (2008). Glycyrrhizin attenuates MPTP neurotoxicity in mouse and MPP-induced cell death in PC12 cells. Korean J. Physiol. Pharmacol. 12, 65-71. doi: 10.4196/kjpp.2008.12.2.65

Kim, Y. M., Kim, H. J., and Chang, K. C. (2015). Glycyrrhizin reduces HMGB1secretion in lipopolysaccharide activated RAW 264.7 cells and endotoxemic mice by p38/Nrf2-dependent induction of $\mathrm{HO}-1$. Int. Immunopharmacol. 26, 112-118. doi: 10.1016/j.intimp.2015.03.014

Lin, F., Pan, L. H., Ruan, L., Qian, W., Liang, R., Ge, W. Y., et al. (2015). Differential expression of HIF-1alpha, AQP-1, and VEGF under acute hypoxic conditions in the non-ventilated lung of a one-lung ventilation rat model. Life Sci. 124, 50-55. doi: 10.1016/j.lfs.2014.12.020

Lin, Q., Li, M., Fang, D., Fang, J., and Su, S. B. (2011). The essential roles of Toll-like receptor signaling pathways in sterile inflammatory diseases. Int. Immunopharmacol. 11, 1422-1432. doi: 10.1016/j.intimp.2011.04.026

Luo, L., Jin, Y., Kim, I. D., and Lee, J. K. (2014). Glycyrrhizin suppresses HMGB1 inductions in the hippocampus and subsequent accumulation in serum of a kainic acid-induced seizure mouse model. Cell. Mol. Neurobiol. 34, 987-997. doi: 10.1007/s10571-014-0075-4

Ma, Y., He, M., and Qiang, L. (2013). Exercise therapy downregulates the overexpression of TLR4, TLR2, MyD88 and NF-kappaB after cerebral ischemia in rats. Int. J. Mol. Sci. 14, 3718-3733. doi: 10.3390/ijms14023718

Miyake, K. (2007). Innate immune sensing of pathogens and danger signals by cell surface Toll-like receptors. Semin. Immunol. 19, 3-10. doi: 10.1016/j.smim. 2006.12.002

Mussi, R. K., Camargo, E. A., Ferreira, T., De Moraes, C., Delbin, M. A., Toro, I. F., et al. (2008). Exercise training reduces pulmonary ischaemia-reperfusioninduced inflammatory responses. Eur. Respir. J. 31, 645-649. doi: 10.1183/ 09031936.00015607

Ogiku, M., Kono, H., Hara, M., Tsuchiya, M., and Fujii, H. (2011). Glycyrrhizin prevents liver injury by inhibition of high-mobility group box1 production by Kupffer cells after ischemia-reperfusion in rats. J. Pharmacol. Exp. Ther. 339, 93-98. doi: 10.1124/jpet.111.182592
Phelan, P., Merry, H. E., Hwang, B., and Mulligan, M. S. (2015). Differential toll-like receptor activation in lung ischemia reperfusion injury. J. Thorac. Cardiovasc. Surg. 149, 1653-1661. doi: 10.1016/j.jtcvs.2015.02.045

Prakash, A., Sundar, S. V., Zhu, Y. G., Tran, A., Lee, J. W., Lowell, C., et al. (2015). Lung ischemia-reperfusion is a sterile inflammatory process influenced by commensal microbiota in mice. Shock 44, 272-279. doi: 10.1097/SHK. 0000000000000415

Salameh, A., and Dhein, S. (2015). Strategies for pharmacological organoprotection during extracorporeal circulation targeting ischemia-reperfusion injury. Front. Pharmacol. 22:296. doi: 10.3389/fphar.2015.00296

Schröfelbauer, B., Raffetseder, J., Hauner, M., Wolkerstorfer, A., Ernst, W., and Szolar, O. H. (2009). Glycyrrhizin, the main active compound in liquorice, attenuates pro-inflammatory responses by interfering with membrane dependent receptor signaling. Biochem. J. 421, 473-482. doi: 10.1042/BJ20082416

Shen, L., Cui, Z., Lin, Y., Wang, S., Zheng, D., and Tan, Q. (2015). Antiinflammative effect of glycyrrhizin on rat thermal injury via inhibition of high-mobility group box 1 protein. Burns 41, 372-378. doi: 10.1016/j.burns. 2014.05.008

Shichita, T., Sakaguchi, R., Suzuki, M., and Yoshimura, A. (2012). Post-ischemic inflammation in the brain. Front. Immunol. 3:132. doi: 10.3389/fimmu.2012. 00132

Shigeoka, A. A., Holscher, T. D., King, A. J., Hall, F. W., Kiosses, W. B., Tobias, P. S., et al. (2007). TLR2 is constitutively expressed within the kidney and participates in ischemic renal injury through both MyD88-depedent and independent pathways. J. Immunol. 178, 6252-6258. doi: 10.4049/jimmunol.178.10. 6252

Wang, J., Nikrad, M. P., Travanty, E. A., Zhou, B., Phang, T., Gao, B., et al. (2012). Innate immune response of human alveolar macrophages during influenza A infection. PLoS ONE 7:e29879. doi: 10.1371/journal.pone. 0029879

Weyker, P. D., Webb, C. A., Kiamanesh, D., and Flynn, B. C. (2013). Lung ischemia reperfusion injury: a bench-to-bedside review. Semin. Cardiothorac. Vasc. Anesth. 17, 28-43. doi: 10.1177/1089253212458329

Yang, H., Hreggvidsdottir, H. S., Palmblad, K., Wang, H., Ochani, M., Li, J., et al. (2010). A critical cysteine is required for HMGB1 binding to Toll-like receptor 4 and activation of macrophage cytokine release. Proc. Natl. Acad. Sci. U.S.A. 107, 11942-11947. doi: 10.1073/pnas.1003893107

Zhang, J., Wu, Y., Weng, Z., Zhou, T., Feng, T., and Lin, Y. (2014). Glycyrrhizin protects brain against ischemia-reperfusion injury in mice through HMGB1TLR4-IL-17A signaling pathway. Brain Res. 25, 176-186. doi: 10.1016/j. brainres.2014.07.002

Zheng, Z. K., Wang, J. J., Hu, H., Jiang, K., Nie, J., Zhang, J., et al. (2013). Shortterm inhalation of nitric oxide inhibits activations of toll-like receptor 2 and 4 in the lung after ischemia-reperfusion injury in mice. J. Huazhong Univ. Sci. Technolog. Med. Sci. 33, 219-223. doi: 10.1007/s11596-013-1100-4

Conflict of Interest Statement: The authors declare that the research was conducted in the absence of any commercial or financial relationships that could be construed as a potential conflict of interest.

Copyright (c) 2017 Fei, Jifeng, Tiantian, Yi and Linghui. This is an open-access article distributed under the terms of the Creative Commons Attribution License (CC BY). The use, distribution or reproduction in other forums is permitted, provided the original author(s) or licensor are credited and that the original publication in this journal is cited, in accordance with accepted academic practice. No use, distribution or reproduction is permitted which does not comply with these terms. 